\title{
Viabilidade econômica do feijoeiro, sob lâminas de irrigação e doses de nitrogênio
}

\author{
Jéssica Carvalho Borges NEVES1, Márcio José de SANTANA¹, André Luís Teixeira FERNANDES², \\ Matheus Pereira de ASSIS 3 , Jan Cornelis VAN KEMPEN³
}

\author{
${ }^{1}$ Instituto Federal de Educação, Ciência e Tecnologia do Triângulo Mineiro, Uberaba, MG, Brasil. \\ 2Universidade de Uberaba, Uberaba, MG, Brasil. \\ ${ }^{3}$ Agrônomo autônomo, Uberaba, MG, Brasil. \\ *E-mail: jessicacbneves@gmail.com \\ (Orcid: 0000-0002-6700-5303; 0000-0003-1985-6880; 0000-0002-2727-3477; \\ 0000-0002-7931-2167; 0000-0003-4569-2576)
}

Recebido em 29/01/2021; Aceito em 02/06/2021; Publicado em 25/06/2021.

\begin{abstract}
RESUMO: A adubação nitrogenada e a irrigação são manejos necessários determinantes para uma boa produtividade. É necessário evitar o uso indiscriminado de adubos e dos recursos hídricos, utilizando técnicas agronômicas que diminuem os impactos socioeconômicos e ambientais. O estudo sobre a viabilidade econômica do feijoeiro é importante para definir gastos futuros, minimizar utilização de recursos naturais e aumentar a produção. O objetivo deste estudo foi avaliar economicamente a produção de feijão (cultivar BRSMG Majestoso) sob diferentes lâminas de irrigação e doses de adubação nitrogenada. O experimento foi conduzido no Instituto Federal do Triângulo Mineiro - Campus Uberaba. Foram utilizados quatro níveis de déficits de água no solo (15, 30, 45\% e um fator sem déficit hídrico) e quatro doses de nitrogênio (50, 100, 150 e $200 \mathrm{~kg} \mathrm{ha}^{-1}$ ), com quatro repetições, em blocos casualizados. Foram realizadas análises da eficiência econômica da lâmina de água e do custo de produção de feijão em função da adubação nitrogenada. Após estudo dos déficits, tanto a lâmina ótima econômica $(446,6 \mathrm{~mm})$ quanto a lâmina física $(448,9 \mathrm{~mm})$ encontradas, foram semelhantes e proporcionaram produtividades similares. O maior rendimento bruto foi de $\mathrm{R} \$ 7.653,99$ quando aplicado $100 \mathrm{~kg} \mathrm{ha}^{-1}$ de nitrogênio.
\end{abstract}

Palavras-chave: água; déficit; adubação; Phaseolus vulgaris L.; economia.

\section{Economic viability of bean, under irrigation blades and nitrogen doses}

\begin{abstract}
Nitrogen fertilization and irrigation are necessary management determinants for good productivity. It is necessary to avoid the indiscriminate use of fertilizers and water resources, using agronomic techniques that reduce socioeconomic and environmental impacts. The study on the economic viability of beans is important to define future spending, minimize the use of natural resources and increase production. The objective of this study was to economically evaluate the production of beans (cultivar BRSMG Majestoso) under different irrigation depths and nitrogen fertilization doses. The experiment was conducted at the Federal Institute of Triângulo Mineiro - Campus Uberaba. Four levels of soil water deficits (15, 30, 45\% and one without water deficit) and four nitrogen doses (50,100, 150 and $200 \mathrm{~kg} \mathrm{ha}^{-1}$ ) were used, with four replications, in randomized blocks. Analyzes of the economic efficiency of the water depth and the cost of bean production were carried out as a function of nitrogen fertilization. After studying the deficits, both the economic optimum blade $(446.6 \mathrm{~mm})$ and the physical blade $(448.9 \mathrm{~mm})$ found were similar and provided similar productivity. The highest gross yield was $\mathrm{R} \$ 7,653.99$ when $100 \mathrm{~kg} \mathrm{ha}^{-1}$ of nitrogen was applied.

Keywords: water; deficit; fertilizing; Phaseolus vulgaris L.; economy.
\end{abstract}

\section{INTRODUÇÃO}

Plantas leguminosas possuem uma variedade de espécies e ampla distribuição mundial, sendo que, o feijão (Phaseolus vulgaris L.) é um dos grãos mais difundidos e consumidos em todo o mundo e presente em todos os continentes. No Brasil, a alta capacidade de adaptação climática e de solos desta leguminosa permite seu cultivo durante todo o ano em todos os estados do país, possibilitando constante oferta do produto no mercado (CONAB, 2019).

Dentre os tratos culturais, a aplicação de nitrogênio e a irrigação se tornam essenciais para uma maior produtividade da cultura. O nitrogênio é geralmente o elemento essencial requerido em maior quantidade pelas plantas. E como elemento essencial sua deficiência impede seu desenvolvimento, ou ainda, limita a sua produção. Sua principal fonte está na atmosfera, na forma de $\mathrm{N}_{2}$.

Todavia, as plantas não o absorvem desta maneira, sendo necessário passar por processos químicos de transformação, para suas formas assimiláveis de nitrato $\left(\mathrm{NO}^{3-}\right)$ e amônio $\left(\mathrm{NH}^{4+}\right)$. O N é absorvido em maior proporção na forma de nitrato, depois do processo denominado nitrificação, tornando disponível para a absorção pelas raízes (MALAVOLTA, 1979).

Para a cultura do feijoeiro, de acordo Soratto et al. (2013), o nitrogênio é exigido em grandes quantidades, pois cerca de 
$50 \%$ da quantidade absorvida será exportada para o grão. Em relação à irrigação, Taiz; Zeiger (2013) relataram que o estresse hídrico modifica processos fisiológicos e morfológicos nas plantas, alterando a célula e inibindo processos de crescimento.

Para a cultura do feijoeiro a água é muito importante, sendo que o estádio de florescimento é crítico em casos de deficiência hídrica. O uso racional de recursos como a água e o adubo nitrogenado, pode acarretar em uma diminuição das despesas do sistema produtivo e o aumento da receita. A análise econômica auxilia nas decisões a serem tomadas, sendo os parâmetros utilizados é a economia dos recursos utilizados, maior produtividade e maior rentabilidade econômica.

Diante desse exposto, o objetivo deste estudo foi avaliar economicamente a cultura do feijão sob diferentes lâminas de irrigação e doses de nitrogênio.

\section{MATERIAL E MÉTODOS}

O estudo foi conduzido no campus Uberaba do Instituto Federal de Educação, Ciência e Tecnologia do Triângulo Mineiro localizado no município de Uberaba - MG, situado a $19^{\circ} 39^{\prime} 14.2^{\prime \prime} \mathrm{S}$ e $47^{\circ} 57^{\prime} 28.8^{\prime \prime} \mathrm{W}$ e de $795 \mathrm{~m}$ acima do nível do mar com pluviosidade média anual de $1600 \mathrm{~mm}$, temperatura média anual de $22,6{ }^{\circ} \mathrm{C}$ e umidade relativa média de 68\% (INMET, 2020). O clima é classificado como AW, tropical quente segundo a classificação de Beck et al. (2018), apresentando inverno frio e seco.

O solo da área experimental foi classificado como Latossolo Vermelho Distrófico, apresentando textura Franco Arenosa (SANTOS et al., 2018), com pH na camada de $0-$ $20 \mathrm{~cm}$ de 5,8 (Tabela 1).

Tabela 1. Características químicas do solo (Uberaba, MG).

Table 1. Soil chemical characteristics (Uberaba, MG).

\begin{tabular}{cc}
\hline Características & Teores \\
\hline $\mathrm{pH} \mathrm{em} \mathrm{CaCl}$ & 5,8 \\
$\mathrm{P}\left(\mathrm{mg} \mathrm{dm}^{-3}\right)$ & 31,12 \\
$\mathrm{~K}\left(\mathrm{mmolcdm}^{-3}\right)$ & 2,96 \\
$\mathrm{Ca}^{2+}\left(\mathrm{mmolcdm}^{-3}\right)$ & 26,2 \\
$\mathrm{Mg}^{2+}\left(\mathrm{mmolcdm}^{-3}\right)$ & 15,7 \\
$\mathrm{Al}^{3+}\left(\mathrm{mmolcdm}^{-3}\right)$ & 0 \\
$\mathrm{H}+\mathrm{Al}\left(\mathrm{mmolcdm}^{-3}\right)$ & 16,0 \\
$\mathrm{SB}\left(\mathrm{mmolcdm}^{-3}\right)$ & 44,86 \\
$\mathrm{~T}\left(\mathrm{mmolcdm}^{-3}\right)$ & 60,86 \\
$\mathrm{~V}(\%)$ & 73,21 \\
M.O. $\left(\mathrm{g} \mathrm{dm}^{-3}\right)$ & 17,8 \\
\hline
\end{tabular}

O experimento foi conduzido em blocos casualizados (DBC), sendo empregado um esquema fatorial $4 \times 4$, constituído por quatro lâminas de irrigação, 15, 30 e 45\% e um com $100 \%$ de reposição de água no solo, de acordo com a necessidade da cultura (definida pelo manejo de irrigação, através do uso de hastes tensiométricas instaladas nas parcelas) e quatro doses de nitrogênio (na fonte ureia com $45 \%$ de N) de 50,100, 150 e 200 kg.ha-1 diferenciadas em cobertura no estágio V4, com a terceira folha trifoliolada se encontra aberta. Foram realizadas quatro repetições. Cada parcela experimental foi constituída de seis linhas de semeadura com três metros de comprimento. As plantas localizadas ao centro da área foram consideradas plantas úteis para coletas de dados (área de $1 \mathrm{~m}^{2}$ ).

A cultivar utilizada no experimento foi a BRSMG Majestoso que apresenta hábito de crescimento indeterminado tipo III e grãos do tipo carioca (EMBRAPA, 2006). O sistema de preparo do solo foi o convencional, realizado com o auxílio de grade niveladora e a abertura dos sulcos por meio de cultivador, com hastes espaçadas a $50 \mathrm{~cm}$. O controle de plantas daninhas foi realizado através de capina manual e o controle de pragas e doenças no feijoeiro foi realizado conforme necessidade, com o uso de agrotóxicos registrados para a cultura.

A colheita foi realizada aos 91 dias após a semeadura. Posteriormente a colheita, os grãos foram pesados e tiveram seu grau de umidade medido, para correção do peso para umidade de 13\% (CARVALHO, 2005) conforme (Equação 1). O peso dos grãos foi expresso em $\mathrm{kg} \mathrm{ha}^{-1}$. A colheita foi realizada manualmente e a determinação da umidade em medidor padrão do IFTM.

$$
P=\frac{P c .(100-U o)}{(100-U i)}
$$

em que: P: peso corrigido (kg ha-1); Pc: peso de campo (kg ha-1); Uo: umidade medida após colheita (\%); U: umidade de correção (13\%).

Os dados climáticos foram obtidos através de um termohigrômetro digital e um pluviômetro modelo Ville de Paris instalados no local do experimento e aferidos pela estação meteorológica automática localizada no IFTM Campus Uberaba (Figuras 1 e 2).

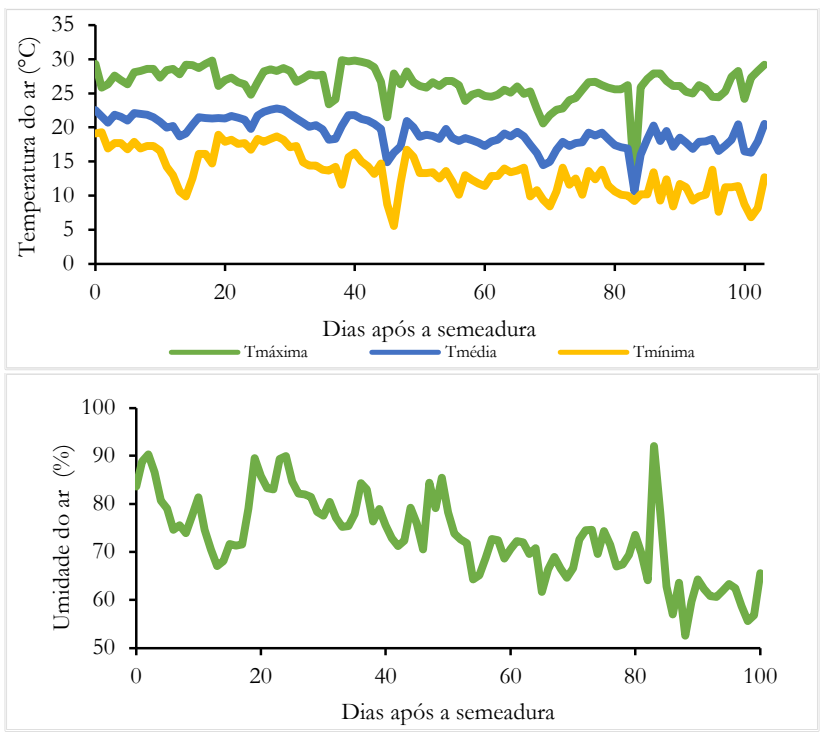

Figura 1. Temperatura e umidade relativa do ar durante o ciclo do feijão, entre abril e agosto de 2017, Uberaba, MG.

Figure 1. Air temperature and humidity relative during the bean cycle, from April to August 2017, Uberaba, MG.

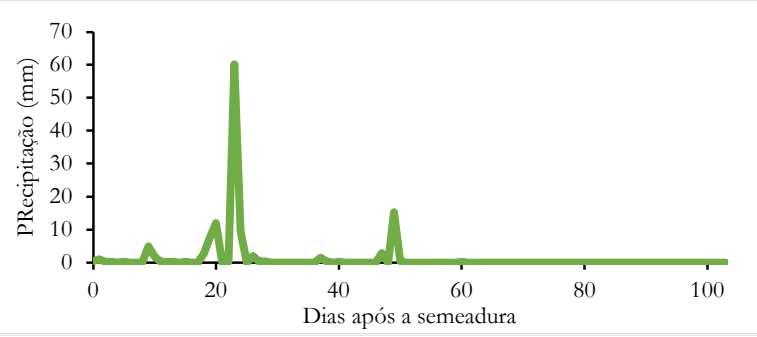

Figura 2. Precipitação durante o ciclo do feijão, entre abril e agosto de 2017, Uberaba, MG.

Figure 2. Precipitation during the bean cycle, from April to August 2017, Uberaba, MG. 
A irrigação foi efetuada por quatro microaspersores instalados nas parcelas, com sobreposição de $50 \%$ instalados a $20 \mathrm{~cm}$ do solo, apresentado a intensidade de aplicação de 7,5 $\mathrm{mm} \mathrm{h}^{-1}$, simulando uma aspersão, elevando os microaspersores do solo. Hastes tensiométricas foram instaladas em cada tratamento para avaliar a tensão de água no solo e umidade. A leitura dos dados foi realizada durante o período da manhã.

Para obtenção da evapotranspiração de referência foi utilizado a equação de Hargreaves (Equação 2).

ETo $=0.0023 X($ Tméd $+17,8) x(\text { Tmáx }- \text { Tmín })^{0,5} \times R a x 0,408(02)$

em que: ETo: evapotranspiração de referência $\left(\mathrm{mm} \mathrm{d}^{-1}\right)$; Tmín: temperatura mínima $\left({ }^{\circ} \mathrm{C}\right)$; Tmáx: temperatura máxima $\left({ }^{\circ} \mathrm{C}\right)$; Tméd: temperatura média $\left({ }^{\circ} \mathrm{C}\right)$; Ra: radiação no topo da atmosfera $(\mathrm{MJ} \mathrm{m}$ $\left.2 \mathrm{~d}^{-1}\right)$.

Os valores de evapotranspiração da cultura no tratamento sem déficit hídrico foram obtidos através das Equação 3 e 4, sendo que as demais lâminas de irrigação obedeceram à mesma proporção.

$$
\begin{aligned}
& \mathrm{ETc}=\mathrm{ETo} \times \mathrm{Kc} \times \mathrm{Ks} \\
& \mathrm{LB}=\mathrm{LL} \div \frac{\mathrm{Cu}}{\mathrm{Ea}}
\end{aligned}
$$

em que: ETc: evapotranspiração da cultura $\left(\mathrm{mm} \mathrm{d}^{-1}\right)$; Kc: coeficiente da cultura (adimensional); Ks: coeficiente de umidade (adimensional); LL: lâmina líquida de irrigação (mm); LB: lâmina bruta de irrigação (mm); Ea: eficiência de aplicação do sistema (decimal) que é a relação entre o volume de água que a planta necessita e o volume de água que o emissor disponibiliza; $\mathrm{Cu}$ : coeficiente de uniformidade do sistema (decimal), através de copos medidores distribuídos na área dos aspersores.

Para o fator de estudo sobre as lâminas de irrigação utilizou-se o modelo de Função de Produção.

O modelo utilizado para o ajuste da função de produção foi um polinômio do segundo grau, conforme Equação 5:

$$
y=f(w)=a+b \cdot x w^{2}+c \cdot x w
$$

em que: y: produtividade (kg há-1); w: lâmina total de água aplicada $(\mathrm{mm}) ; \mathrm{a}, \mathrm{b}$ e c: parâmetros da equação.

Para a composição do custo da irrigação a ser empregado na análise econômica, tomou-se por base o sistema de pivô central por expressar a realidade dos produtores da região, com especificações constantes da Tabela 2.

O custo do sistema de irrigação foi de $\mathrm{R} \$$ 520.000,00, sendo o custo por ha de $\mathrm{R} \$ 8.665,22$. O valor residual foi estimado em $20 \%$ do valor da aquisição, o que equivale a $\mathrm{R} \$$ 104.000,00. A depreciação do sistema foi calculada conforme Equação 6:

$$
D p=\frac{\begin{array}{c}
(V c-V r) \\
V u
\end{array}}{}
$$

em que: Dp: depreciação do sistema (R\$); Vc: valor de compra do sistema (R\$); Vr: valor residual do sistema (R\$); Vu: vida útil do sistema (anos).

Considerando que o equipamento será utilizado por dois ciclos em cada ano e a vida útil de 10 anos, a depreciação é de $\mathrm{R} \$ 346,60$ por hectare por ciclo.
A manutenção e a operação do sistema equivalem a $2 \%$ do valor de aquisição, o que representa $\mathrm{R} \$ 86,65$ por hectare e ciclo de produção.

O custo da energia para bombeamento varia de acordo com a classe de consumidor e tipo de contrato. Consideraram-se no presente estudo, a classe consumo rural e o grupo de tensão A, em função do motor utilizado pelo sistema de irrigação (rede trifásica de $380 \mathrm{~V}$ ). Foram considerados a demanda de potência $(\mathrm{kW})$ e o consumo de energia $\left(\mathrm{kW} \mathrm{h}^{-1}\right)$; adotou-se, para a análise a tarifa horosazonal, considerando o horário fora de ponta (HFP) e o horário fora de ponta/noturno $(\mathrm{HFP} / \mathrm{N})$, cujo valor representa $22 \%$ do valor cobrado pelo HFP (todos no período seco). O consumo de energia (C), a demanda de potência (D) e o custo final (CF) de energia ( $R$ \$) foram calculados com as Equações 7,8 e 9 . A potência $(\mathrm{P})$ é dada em cv.

$$
\begin{gathered}
C=2,64+0,8 \cdot P \\
D=\frac{P \cdot 0,736}{\eta} \\
C F=\frac{\left(D_{c} \cdot T_{d}\right)+\left(C_{f p} \cdot T_{f p} \cdot H_{f p}\right)+\left(C_{f p n} \cdot T_{f p n} \cdot H_{f p n}\right)}{0,82}
\end{gathered}
$$

em que: $\eta$ : rendimento, decimal; $D_{i}$ demanda total contratada, $\mathrm{kW}$; $T_{d:}$ tarifa de demanda, $\mathrm{R} \$ \mathrm{~kW} ; C_{\not p}$ : consumo de energia em horário fora de ponta, $\mathrm{kW} \mathrm{h}^{-1} ; T_{f p}$ : tarifa de consumo em horário fora de ponta, $\mathrm{R} \$ \mathrm{~kW} \mathrm{~h}{ }^{-1} ; H_{p p}$ : horas utilizadas no horário fora de ponta, h; $C_{f p n}$ : consumo de energia em horário fora de ponta/noturno, $\mathrm{kW} \mathrm{h}$ 1; $T_{f p n}$ : tarifa de consumo em horário fora de ponta/noturno, $\mathrm{R} \$ \mathrm{~kW}$ $\mathrm{h}^{-1} ; H_{p p n}$ : horas utilizadas no horário fora de ponta/noturno, $\mathrm{h}$.

Tabela 2. Características do sistema pivô central utilizado nos cálculos dos custos da energia.

Table 2. Characteristics of the central pivot system used in energy cost calculations.

\begin{tabular}{lc}
\hline \multicolumn{1}{c}{ Item } & Descrição \\
\hline Marca & Valley \\
Modelo & 4871-8000-VSL/8-1.007 \\
Propulsão & Elétrica \\
Número de torres & 8 \\
Raio total irrigado & $437,07 \mathrm{~m}$ \\
Área circular irrigada & $60,01 \mathrm{ha}$ \\
Altura livre entre torres & $2,74 \mathrm{~m}$ \\
Precipitação diária (lâmina bruta) & $8,0 \mathrm{~mm} \mathrm{dia}^{-1}$ \\
Período (rele a 100\%) & $9,24 \mathrm{~h}$ \\
Lâmina por percurso (a 100\%) & $3,52 \mathrm{~mm}$
\end{tabular}

\section{Composição}

4 lances médio e 4 lances longos, com balanço de 20 $\mathrm{m}$, com spray final, sem canhão final.

Aspersores

Vazão total

Comprimento até a última torre

Pressão no final da tubulação

Perda de carga total

Altura manométrica total

Vazão por área

Comprimento da tubulação

I Wob com peso, com tubo de descida flexível

$228,61 \mathrm{~m}^{3} \mathrm{~h}^{-1}$

$411,69 \mathrm{~m}$

$13,0 \mathrm{~atm}$

$5,8322 \mathrm{mca}$

$70,66 \mathrm{mca}$

$3,81 \mathrm{~m}^{3} \mathrm{~h}^{-1} \mathrm{ha}^{-1}$ $432,57 \mathrm{~m}$

Dados cedidos pela Valmont, Uberaba, MG. Novembro, 2020. 
Para o cálculo da energia de bombeamento, utilizou-se a lâmina aplicada no tratamento $100 \%$ (472 mm). Considerouse o pivô a $60 \%$ de velocidade no per centímetro a cada irrigação, com um total de 15,4 h, sendo 9 h de irrigação no $\mathrm{HFP} / \mathrm{N}$ e $6,4 \mathrm{~h}$ no HFP. A potência no eixo calculada foi de $80,24 \mathrm{cv}$ e a comercial de $100 \mathrm{cv}$, com rendimentos de motor e bomba de 0,9 e 0,73, respectivamente. Os valores de tarifação para novembro de 2020 foram: demanda HFP de $\mathrm{R} \$ 3,70$ e consumo HFP período seco $\mathrm{R} \$ 0,50552 \mathrm{~kW} \mathrm{~h}{ }^{-1}$ e $\mathrm{R} \$ 0,3282 \mathrm{~kW} \mathrm{~h}^{-1}$ para HFP noturno.

Substituindo-se todos os valores citados anteriormente nas Equações 7, 8 e 9 tem-se um custo de bombeamento de $\mathrm{R} \$ 1.128,71$ por ha e ciclo de cultivo.

Não foi considerado o custo para o volume de água utilizado, sendo a captação considerada pública ou de uso do produtor.

Os custos operacionais efetivos (variáveis) foram compostos apenas pela energia de bombeamento e manutenção. A margem bruta foi dada por $\mathrm{MB}=\mathrm{Py} \times \mathrm{Y}$ custo operacional efetivo.

O preço de venda do feijão verde foi a partir da média dos meses no ano de 2019 sendo de $\mathrm{R} \$ 2,91$ por quilo.

$\mathrm{O}$ custo do fator água (energia + manutenção) foi de $\mathrm{R} \$$ 2,39 por mm de lâmina aplicada.

Considerando que o lucro é máximo quando a primeira derivada do rendimento em relação à lâmina total de água for igual à relação entre fator e produto (Equação 10), tem-se:

$$
\frac{\partial Y}{\partial W}=b+2 c W=\frac{P w}{P y}
$$

Considerou-se como lâmina de água economicamente ótima aquela que conduziu ao rendimento com máxima margem bruta. Dessa forma derivou-se a estimativa das funções de produção, obtendo-se o valor do PFMa e, em seguida, igualou-se à relação preço da água e do produto (Equação 11).

$$
\frac{\partial Y}{\partial W}=162,5-0,362 W=\frac{P w}{P y}
$$

Optou-se para a avaliação econômica das doses de nitrogênio, utilizando planilhas de custos de produção para cada tratamento.

A análise econômica para os tratamentos de aplicação de nitrogênio foi baseada no modelo de custo de produção do (AGRIANUAL, 2019). Foram gerados quatro custos de produção (um por tratamento).

Foram considerados os custos da ureia e sua aplicação (a lanço). O preço da ureia foi de $\mathrm{R} \$ 3,64 \mathrm{~kg}$. Comparou-se o incremento de produtividade, o custo de produção, o acréscimo da receita bruta e o acréscimo da receita líquida. Entende-se por incremento de produtividade a diferença entre a produtividade de grãos correspondente a cada um dos tratamentos. O acréscimo de receita bruta foi determinado pelo acréscimo de produtividade multiplicado pelo preço de venda do feijão. O acréscimo de receita líquida foi obtido pela diferença entre o acréscimo de receita bruta e os custos da adubação nitrogenada. Para a variável produtividade foi realizada análise de variância pelo teste $\mathrm{F}$ a $5 \%$ de probabilidade e regressão para análise das médias.

\section{RESULTADOS}

$\mathrm{Na}$ Tabela 3 estão apresentados os valores médios de tensão de água no solo e a lâmina total aplicada em cada tratamento. As tensões de água no solo média variaram de 12,83 a $16,32 \mathrm{kPa}$. Os déficits foram feitos a partir da lâmina diária total, retirando a porcentagem do déficit.

Tabela 3. Tensão de água no solo e lâminas aplicadas no experimento, Uberaba, MG.

Table 3. Soil water tension and applied blades in the experiment, Uberaba, MG.

\begin{tabular}{ccc}
\hline $\begin{array}{c}\text { Déficit Hídrico } \\
(\%)\end{array}$ & $\begin{array}{c}\text { Tensão de água no } \\
\text { solo }(\mathrm{kPa})\end{array}$ & $\begin{array}{c}\text { Lâmina aplicada } \\
(\mathrm{mm})\end{array}$ \\
\hline 0 & 12,83 & 472 \\
15 & 13,56 & 433 \\
30 & 16,32 & 394 \\
45 & 14,17 & 354 \\
\hline
\end{tabular}

Em relação aos dados climáticos, somando-se a precipitação durante a condução do experimento, foram verificadas as lâminas de 472, 433, 394 e $354 \mathrm{~mm}$ aos respectivos déficits de $0,15,30$ e $45 \%$. As umidades relativas do ar mínima, média e máxima durante o desenvolvimento da cultura foram de 52,62\%; $72,80 \%$ e $92,06 \%$, respectivamente. A temperatura no período de desenvolvimento da cultura variou de $5,60^{\circ} \mathrm{C}$ a $29,90^{\circ} \mathrm{C}$, sendo que a temperatura média durante a condução do experimento foi de $19,1^{\circ} \mathrm{C}$. O valor de temperatura recomendado para o pleno desenvolvimento do feijoeiro é de $29^{\circ} \mathrm{C}$ durante o dia e $21^{\circ} \mathrm{C}$ durante a noite.

As doses de nitrogênio não influenciaram na produtividade do feijoeiro, já os déficits influenciaram a produtividade e esses dados foram significativos a $0,05 \%$. Não houve interação significativa entre os déficits hídricos e as doses de nitrogênio, sendo avaliados seus comportamentos separadamente (Tabela 3).

Tabela 4. Resumo do quadro de Análise de Variância para a variável: Produtividade de grãos (Prod.), Uberaba, MG.

Table 4. Summary of the Analysis of Variance table for the variable: Grain yield (Prod.), Uberaba, MG.

\begin{tabular}{ccc}
\hline $\mathrm{FV}^{1}$ & $\mathrm{GL}^{2}$ & Prod. \\
\hline Déficit (D) & 3 & $0,000^{4}$ \\
Doses de & 3 & $0,9870^{\mathrm{ns}}$ \\
D X N & 9 & $0,6983^{\mathrm{ns}}$ \\
Média geral & - & $4.174,24 \mathrm{~kg} \mathrm{ha}^{-1}$ \\
$\mathrm{CV}^{3}(\%)$ & - & 21,23 \\
Erro & 45 & - \\
\hline
\end{tabular}

A produtividade foi influenciada pelas lâminas de irrigação, dando efeito significativo aos tratamentos. Maiores produtividades foram alcançadas quando o déficit hídrico atingiu 9,72\%. Observa-se que a produtividade decresceu quando a lâmina de água ultrapassou os $450 \mathrm{~mm}$.

Os produtos físicos marginais (PFMa) foram obtidos a partir da função de produção (Figura 3) gerada a equação Y. Derivando a equação da Figura 3, obtivemos as lâminas física econômica e ótima econômica e também as produtividades físicas econômica e ótimas econômica.

A lâmina física (448,9 mm) e a lâmina econômica (446,6 $\mathrm{mm}$ ) foram semelhantes e proporcionaram valores de produtividades aproximadas, que foram $4.859,72 \mathrm{~kg} e$ $4.858,76 \mathrm{~kg}$ respectivamente (Tabela 5). A diferença entre as lâminas física e ótima econômica foi $2,3 \mathrm{~mm}$, ao preço de $\mathrm{R} \$$ 
2,39 o mm de água, o custo dessa diferença foi de $\mathrm{R} \$ 5,49$. As diferenças dessas produtividades foram de $0,96 \mathrm{~kg}$. Considerando o valor médio do quilo do feijão de $\mathrm{R} \$ 2,91$, o custo da diferença seria de $\mathrm{R} \$ 2,79 \mathrm{~kg}$.

Para a análise econômica das doses de nitrogênio, o maior rendimento bruto do feijão se deu quando foi incorporado $100 \mathrm{~kg} \mathrm{ha}^{-1}$ de nitrogênio. O tratamento com $150 \mathrm{~kg} \mathrm{ha}^{-1} \mathrm{de}$ nitrogênio proporcionou menor produtividade do feijão de $4.170,00 \mathrm{~kg}$, foi um dos custos de produção mais onerosos $(\mathrm{R} \$ 4.786,33)$ e também com um dos menores rendimento bruto $(\mathrm{R} \$ 7.348,37)$ (Tabela 6).

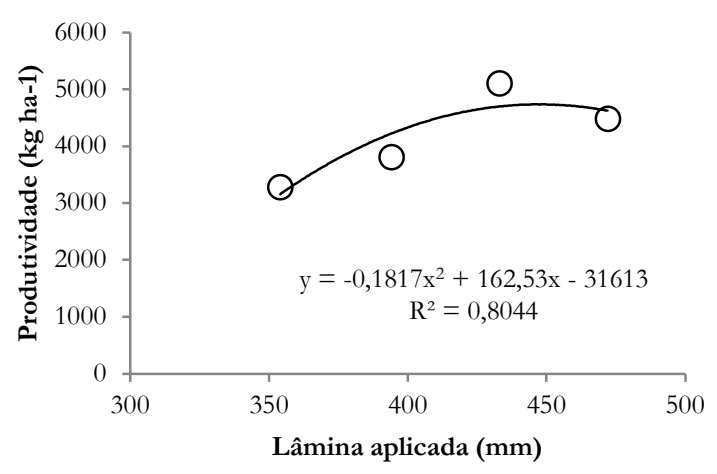

Figura 3. Produtividade do feijão em função das lâminas aplicadas. Figure 4. Bean yield as a function of applied blades.

Tabela 5. Lâminas que proporcionaram máxima produtividade física estimada, produtividade física máxima estimada, lâminas que proporcionaram máxima produtividade econômica, produtividade economicamente ótima e porcentagem que a lâmina ótima econômica representa da lâmina física estimada.

Table 5. Blades that provided maximum estimated physical productivity, estimated maximum physical productivity, blades that provided maximum economic productivity, economically optimal productivity and percentage that the economic optimal blade represents of the estimated physical blade.

\begin{tabular}{ccccc}
\hline L.F. $(\mathrm{mm})$ & $\begin{array}{c}\text { P.F. } \\
\left(\mathrm{kg} \mathrm{ha}^{-1}\right)\end{array}$ & $\begin{array}{c}\text { L.O. } \\
(\mathrm{mm})\end{array}$ & $\begin{array}{c}\text { P.O. } \\
\left(\mathrm{kg} \mathrm{ha}^{-1}\right)\end{array}$ & $\%$ L.F. \\
\hline 448,9 & $4.859,72$ & 446,6 & $4.858,76$ & 99,98 \\
\hline
\end{tabular}

L.F. = lâmina física estimada; P.F. = produtividade física estimada; L.O. = lâmina ótima; P.O. = produtividade ótima e \%L.F. $=$ porcentagem que a lâmina ótima representa da lâmina física.

Tabela 6. Valores para custo de produção e lucro gerado por cada tratamento do feijão, por hectare. Uberaba, MG.

Table 6. Values for production cost and profit generated by each bean treatment, per hectare. Uberaba, MG.

\begin{tabular}{cccc}
\hline $\begin{array}{c}\text { Doses de N } \\
\left(\mathrm{kg} \mathrm{ha}^{-1}\right)\end{array}$ & $\begin{array}{c}\text { Produtividade } \\
\left(\mathrm{kg} \mathrm{ha}^{-1}\right)\end{array}$ & $\begin{array}{c}\text { Custo de } \\
\text { Produção } \\
\left(\mathrm{R} \$ \mathrm{ha}^{-1}\right)^{*}\end{array}$ & $\begin{array}{c}\text { Rendimento } \\
\text { bruto } \\
\left(\mathrm{R} \$ \mathrm{ha}^{-1}\right)\end{array}$ \\
\hline 50 & $4.108,20$ & $4.422,33$ & $7.532,53$ \\
100 & $4.212,48$ & $4.604,33$ & $7.653,99$ \\
150 & $4.170,00$ & $4.786,33$ & $7.348,37$ \\
200 & $4.205,00$ & $4.968,33$ & $7.268,22$ \\
\hline
\end{tabular}

*valores baseados no Agrianual 2019, para um hectare plantado.

Para o ano de 2019, o custo da ureia foi de $\mathrm{R} \$ 3,64 \mathrm{~kg}$, valor muito a cima dos anos anteriores. O quilo do feijão para o ano de 2019, nas regiões produtoras de Minas Gerais foi de $\mathrm{R} \$ 2,91$, sendo que o quilo do feijão já estava sob um valor maior do que a média dos anos anteriores. Doses altas de nitrogênio aumentam o custo de produção do feijão, sendo necessária a utilização de análise de dados para verificar a viabilidade da dose.

\section{DISCUSSÃO}

Ao analisar os dados, nota-se que a tensão de água no solo variou e não foi suficiente para avaliar as lâminas de água, sendo necessário fazer a análise econômica. Dentre as tensões de água no solo (30, 40, 50, 60 e $70 \mathrm{kPa})$ estudadas por Brito et al. (2015), a tensão que promoveu maior incremento na produtividade do feijão cv. BRSMG Majestoso foi a de $30 \mathrm{kPa}$. Sobre o nitrogênio, Pacheco et al. (2016) e Guimarães et al. (2017) observaram que as doses de nitrogênio não apresentaram efeito significativos para a cultura.

Infere-se que a fixação biológica de nitrogênio está sendo eficiente e está suprindo as necessidades da planta sobre esse nutriente. Pacheco et al. (2016) e Guimarães et al. (2017) também observaram que o nitrogênio não apresentou resultados significativos para a cultura, sem diferença estatística.

O déficit hídrico foi significativo, com aumento expressivo da produtividade. Avaliando os níveis de déficit hídrico no solo de 25, 50, 75, 100, 125, e 150\%, Aleman; Mingnacca (2015) concluíram que a cultivar Pérola produziu mais no nível de $75 \%$ de água no solo, sendo benéfico para a cultura. Em trabalho conduzido por Sales et al. (2017), foi verificado que a maior produtividade da cultivar BRSMG Majestoso foi de 3.422,19 $\mathrm{kg} \mathrm{ha}^{-1}$, com uma lâmina de 226,96 $\mathrm{mm}$, sendo esta lâmina menor cerca de $29 \%$ da lâmina de $319,68 \mathrm{~mm}$ que foi a maior utilizada.

Torres et al. (2013), avaliando diferentes coberturas do solo e lâminas de irrigação (40, 70, 100, 130 e 160\% da evapotranspiração diária), também se verificou uma maior produtividade de grãos quando utilizado a reposição de $100 \%$.

Comparando os resultados obtidos nesse experimento com os resultados encontrados na literatura, nota-se que déficits pequenos ocasionam produtividades semelhantes à produtividades com lâminas de 100\% de reposição de água no solo. Isso mostra que pequenos déficits podem gerar economia do uso da água e consequentemente do uso de energia elétrica.

Como o preço do quilo do feijão ficou aproximado do valor do custo do valor de água, sendo alguns centavos de diferença $(\mathrm{R} \$ 0,52)$, a porcentagem de que a lâmina ótima econômica representa da lâmina física estimada ficou próxima de $100 \%$. Isso mostra que tanto a lâmina ótima quanto a lâmina física podem ser utilizados e que proporcionaram produtividades semelhantes, sendo necessário utilizar critérios de racionalidade de recursos.

A melhor eficiência no uso da água para Osti et al. (2019), foi pela a lâmina ótima de $522,17 \mathrm{~mm}$. A lâmina considerada ótima economicamente reduz gastos com a quantidade de água consumida, reduz os custos das lâminas, otimizando lucros, com equilíbrio financeiro e ambiental (SILVA et al., 2013).

Silva et al. (2019) verificaram que a máxima produção de $1.760 \mathrm{~kg} \mathrm{ha}^{-1}$ foi com $204 \mathrm{~mm}$ e com o valor da saca de $60 \mathrm{~kg}$ de $\mathrm{R} \$ 70,00$. As produtividades máximas econômicas de $1.790 \mathrm{~kg} \mathrm{ha}^{-1}$ e $1.810 \mathrm{~kg} \mathrm{ha}^{-1}$ foram atingidas com $221 \mathrm{~mm}$ e $232 \mathrm{~mm}$ de água e com valores de saca de $\mathrm{R} \$ 90,00$ e $\mathrm{R} \$$ 110,00 . 
Sobre o custo de produção do feijão sob as doses de nitrogênio, comparou-se os tratamentos de $50 \mathrm{~kg} \mathrm{ha}^{-1} \mathrm{com} o$ $200 \mathrm{~kg} \mathrm{ha}^{-1}$ a diferença de produtividade chega a $96,8 \mathrm{~kg} \mathrm{ha}^{-1}$ e a diferença do custo de produção são de $\mathrm{R} \$ 546,00$ por hectare. Multiplicando esses valores, a diferença de produtividade em 60 ha é de $5.808,00 \mathrm{~kg}$ e o valor do nitrogênio de $\mathrm{R} \$ 32.760,00$. O custo do nitrogênio impactou o custo de produção da cultura, comparando a dose de $50 \mathrm{~kg}$ $\mathrm{ha}^{-1} \mathrm{com}$ a dose de $200 \mathrm{~kg} \mathrm{ha}^{-1}$. Para Rosa et al. (2020), a dose de $240 \mathrm{~kg} \mathrm{ha}^{-1}$ de $\mathrm{N}$ em cobertura, resultou na maior receita líquida e consequentemente na maior viabilidade econômica.

Fiorentin et al. (2012) observaram que, na sucessão com milho-grão exclusivo, onde o aumento da produtividade pela aplicação de $\mathrm{N}$ em cobertura foi crescente, a única margem bruta positiva de ganho na venda dos grãos de feijão foi alcançada com o uso da dose de $160 \mathrm{~kg} \mathrm{ha}^{-1}$ de N, o que, segundo os autores, demonstra ser indispensável a utilização de elevadas doses de $\mathrm{N}$ em cobertura, para o feijoeiro cultivado em sucessão ao milho-grão.

Entretanto, para Oliveira et al. (2019) a cultura tem baixa eficiência na absorção de $\mathrm{N}$ pelas raízes e exige em média 100 $\mathrm{kg} \mathrm{ha}^{-1}$ para suprir suas necessidades nutricionais.

\section{CONCLUSÕES}

O maior rendimento bruto foi proporcionado com a aplicação de $100 \mathrm{~kg} \mathrm{ha}^{-1}$ de nitrogênio. A dose de $150 \mathrm{~kg} \mathrm{ha}^{-1}$ de nitrogênio promoveu o menor rendimento bruto e o segundo custo de produção mais oneroso e a dose de $200 \mathrm{~kg}$ ha- ${ }^{1}$ apresentou maior custo de produção e menor rendimento bruto.

A lâmina de água que proporcionou maior produtividade foi de $448,9 \mathrm{~mm}$.

\section{AGRADECIMENTOS}

Ao Instituto Federal do Triângulo Mineiro pelo apoio durante a conclusão do Mestrado.

\section{REFERÊNCIAS}

AGRIANUAL. Anuário da agricultura brasileira. 24 ed. São Paulo: FNP, 2019.

ALEMAN, C. C.; MIGNACCA, F. A. Avaliações morfofisiológicas do feijão Pérola irrigado. Colloquium Agrariae, v. 11, n. 2, p. 19-24, 2015.

BECK, H. E.; ZIMMERMANN, N. E.; MCVICAR, T. R.; VERGOPOLAN, N.; BERG, A.; WOOD, E. F. Present and future Köppen-Geiger climate classification maps at 1-km resolution. Scientific Data, p. 1-12, 2018.

BRITO, R. R.; FILHO, H. G.; SAAD, J. C. C.; RIBEIRO, V. Q.; OLIVEIRA, S. R. M. Critérios de manejo na irrigação do feijoeiro em solo de textura arenosa. Irriga, Botucatu, v. 20, n. 2, p. 334-347, 2015.

CARVALHO, J. A. Coeficiente de cultura, avaliação econômica da produção e análise do crescimento da cenoura (Daucuscarota L.) irrigada. Viçosa, 1995. 77 p. Tese (Doutorado em Engenharia Agrícola) Universidade Federal de Viçosa, 1995.

COMPANHIA NACIONAL DE ABASTECIMENTO (CONAB). Acompanhamento da safra brasileira de grãos. v. 6, n. 7, p. 1-119, 2019.

EMBRAPA ARROZ E FEIJÃO. BRSMG Majestoso. Feijoeiro comum de grão tipo carioca. Santo Antônio de Goiás, GO. 2006. 2p. (Folder)
FIORENTIN, C. F.; LEMOS L. B., JARDIM, C. A.; FORNASIERI FILHO, D. Adubação nitrogenada de cobertura no feijoeiro de inverno-primavera em três sistemas de cultivo. Londrina: Semina: Ciências Agrárias, Londrina, v. 33, n. 1, p. 2825-2835, 2012.

GUIMARÃES, R. A. M.; BRAZ, A. J.B. P.; SIMON, G. A.; FERREIRA, C. J. B.; BRAZ, G. B. P.; SILVEIRA, P. M. Resposta de cultivares de feijoeiro a adubação nitrogenada em diferentes estádios fenológicos. Global Science And Technology, Rio Verde, v. 10, n. 1, p.136148, 2017.

INSTITUTO NACIONAL DE METEOROLOGIA INMET. Dados da estação automática de Uberaba. http://www.inmet.gov.br/. 04 Dez. 2020.

KOLLING, D. F.; OZELAME, O. Utilização de nitrogênio e enxofre em cobertura no feijão preto safrinha. Scientia Agraria, v. 18, n. 4, p. 204-211, 2017. DOI: http://dx.doi.org/10.5380/rsa.v18i4.51205.

MALAVOLTA, E. ABC da adubação. Piracicaba: Editora Agronômica Ceres, 1979. 255p.

OLIVEIRA, R. A.; BUSO, W. H. D.; GOMES. L. L.; MARTINS, P. H. M.; RODOVALHO, R. S.; OLIVEIRA, H. F. E de. Application of controlledrelease nitrogen fertilizer in irrigated common bean crops. Comunicata Scientiae, Bom Jesus, v. 10, p. 158167, 2019. DOI: https://doi.org/10.14295/cs.v10i1.2995.

OSTI, A. M.; DALLACORT, R.; TIEPPO, R. C.; GRZEBIELUCKAS, C.; CONCEIÇÃO, A. M. Rentabilidade do milho e do feijão submetido a diferentes lâminas de irrigação em Mato Grosso. Revista de Economia e Sociologia Rural, Brasília, v. 57, n. 4, p. 505-518, 2019. https://doi.org/10.1590/18069479.2019.186329.

PACHECO, A.; LOPES, A. S.; OLIVEIRA, G. Q.; FRANÇA, A.; SILVA, L. E. Diferentes métodos de manejo de irrigação e doses de adubação nitrogenada nos componentes de produtividade e eficiência no uso da água. Agrarian, Dourados, v. 9, n. 33, p. 263-273, 2016.

ROSA, W. B.; DUARTE JÚNIOR, J. B.; COSTA, A. C. T. DA.; LANA, M. C.; QUEIROZ, S. B.; PEREGO, I.; ABRAÃO, P. C. Desempenho agronômico e viabilidade econômica da adubação nitrogenada e molíbdica no feijão comum. Brazilian Journal of Development, São José dos Pinhais, v. 6, n. 9, p. 65815-65831, 2020. DOI: https://doi.org/10.34117/bjdv6n9-129

SALES, R. A.; AMBROZIM, C. S.; POSSE, R. P.; OLIVEIRA, E. C.; POSSE, S. P. Índice de satisfação das demandas de água e produtividade do feijão em diferentes lâminas de irrigação em Colatina - ES. Energia na Agricultura, Viçosa, v. 32, n. 1, p. 81-87, 2017. DOI: https://doi.org/10.17224/EnergAgric.2017v32n1p8187.

SANTOS, H. G.; JACOMINE, P. K. T.; ANJOS, L. H. C.; OLIVEIRA, V. A.; LUMBRERAS, J. F.; COELHO, M. R.; ALMEIDA, J. A.; CUNHA, T. J. F.; OLIVEIRA, J. B. Sistema brasileiro de classificação de solos. 3 ed. Brasília: Embrapa Informação Tecnológica, 2018. 355p.

SILVA, S.; SILVA, C. S. da.; SOUZA, J. E.; SOUSA, A. C. P.; ARAÚJO, E. R. Uso econômico da água para o feijão caupi na região do sertão alagoano. Revista Brasileira de Agrotecnologia, Pombal, v. 9, n. 1, p. 7-13, 2019. DOI: https://doi.org/10.18378/rebagro.v9i1.6452 
SILVA, V. P. R.; ALEIXO, D. O.; DANTAS NETO, J. MARACAJÁ, K. F. B.; ARAÚJO, L. E. de. Uma medida de sustentabilidade ambiental: Pegada hídrica. Revista Brasileira de Engenharia Agrícola e Ambiental, Campina Grande, v. 17, n. 1, p. 100-105, 2013. DOI: https://doi.org/10.1590/S1415-43662013000100014

SORATTO, R. P.; FERNANDES, A. M.; SANTOS, L. A dos.; JOB, A. L. G. Nutrient traction and exportation by common bean cultivars under different fertilization levels: I - Macronutrients. Revista Brasileira de Ciência do Solo, Viçosa, v. 37, n. 4, p. 1027-1042, 2013. DOI: https://doi.org/10.1590/S0100-06832013000400020.

TORRES, J. L. R.; SANTANA, M. J.; PIZOLATO NETO, A.; PEREIRA, M. G.; VIEIRA, D. M. S. Produtividade de feijão sobre lâminas de irrigação e coberturas de solo.
Bioscience Journal, Uberlândia, v. 29, n. 4, p. 833-841, 2013.

VIEIRA, C. Adubação Mineral e Calagem. In: VIEIRA, C.; PAULA JUNIOR, T. J., BORÉM, A. (Eds.). Feijão: aspectos gerais e cultura no Estado de Minas. Viçosa: UFV, 1998. p.123-152.

TAIZ, L.; ZEIGER, E. Fisiologia vegetal. Porto Alegre: Artmed, 2013. 918p.

VITTI, A. C.; TRIVELIN, P. C. O.; GAVA, G. J. C.; PENATTI, C. P.; BOLOGNA, I. R.; FARONI, C. E.; FRANCO, H. C. J. Produtividade da cana de açúcar relacionada ao nitrogênio residual da adubação e do sistema radicular. Pesquisa Agropecuária Brasileira, Brasília, v. 42, n. 2, p. 249-256, 2007. DOI: http://dx.doi.org/10.1590/S0100-204X2007000200014. 